All authors: The University of Texas MD Anderson Cancer Center, Houston, TX. Published online ahead of print at www.jco.org on May 5, 2014

Supported in part by Grants No. RO1CA1R010162-01A1, R01CA1222292-01, and R01CA124481-01 from the National Institutes of Health (E.B.).

Authors' disclosures of potential conflicts of interest and author contributions are found at the end of this article.

Corresponding author: Eduardo Bruera, MD, Department of Palliative Care \& Rehabilitation Medicine, Unit 1414, The University of Texas MD Anderson Cancer Center, 1515 Holcombe Blvd, Houston, TX 77030; e-mail: ebruera@mdanderson.org

(c) 2014 by American Society of Clinical Oncology

0732-183X/14/3216w-1640w/\$20.00 DOI: 10.1200/JCO.2013.52.2508

\title{
A Personalized Approach to Assessing and Managing Pain in Patients With Cancer
}

David Hui and Eduardo Bruera

$$
\begin{array}{llllllll}
\text { A } & \text { B } & \text { S } & \text { T } & \text { R } & \text { A } & \text { C } & \text { T }
\end{array}
$$

Pain is one of the most common and distressing symptoms in patients with cancer. In this review, we discuss an evidence-based approach to personalized pain assessment and management. Recent insights into the pain expression pathway have led to a paradigm shift in pain management, allowing clinicians to deliver personalized treatments tailored to the individual's needs. Personalized pain management begins with systematic screening, followed by comprehensive pain assessment. Impeccable characterization of pain informs its etiology and the mechanism to guide treatment choices. Identification of modulators of pain expression such as psychological distress, alcoholism, substance use, and delirium allow clinicians to further tailor treatment recommendations. Documentation of a personalized pain goal provides an individualized response criterion. A multidimensional treatment plan is then formulated targeting the pain mechanism, etiologic factors, and modulators. Finally, longitudinal monitoring customized to the individual's needs allows clinicians to improve adherence and, ultimately, to optimize pain control over time.

\section{J Clin Oncol 32:1640-1646. (C) 2014 by American Society of Clinical Oncology}

\section{INTRODUCTION}

Pain is one of the most common and distressing symptoms in patients with cancer, affecting $49 \%$ to $57 \%$ of patients with curable cancer and $56 \%$ to $75 \%$ of patients with advanced disease. ${ }^{1-3}$ Poorly controlled pain can significantly impair function, appetite, sleep, mood, and quality of life. Importantly, approximately $90 \%$ of patients can achieve good pain control by following pain treatment guidelines. ${ }^{4,5}$ However, pain management remains suboptimal in the busy oncology setting, which represents many missed opportunities to improve patients' quality of life. ${ }^{6}$

Impeccable management of pain begins with appropriate assessment, which includes documentation of pain characteristics, determination of pain mechanism, identification of modulating factors, clarification of a personalized pain goal, and regular reassessments over time. In this article, we discuss an evidence-based approach to personalized pain assessment and management.

\section{PAIN CHARACTERISTICS}

Comprehensive pain assessment involves a careful inquiry of pain characteristics. In addition to pain intensity, location, medical treatments, number of episodes, onset, position, quality, radiation, severity, and triggers (a useful mnemonic is LMNOPQRST) can provide important diagnostic clues to the mechanism of pain and inform personalized management.

Pain intensity as reported by the patient is the gold standard for pain assessment. ${ }^{7}$ The three most commonly used scales are the numeric rating scale ( 0 to 10 [0, no pain; 10 , worst possible pain]), the visual analog scale ( 0 to $100 \mathrm{~mm}$; [0, no pain; 100 , worst possible pain]), and the categorical scale (none, mild, moderate, severe). ${ }^{7}$ In general, a numeric score of 1 to 3,4 to 6 , and 7 to 10 corresponds to mild, moderate, and severe pain, respectively. It is important to pay attention to the time frame because the answer may vary, depending on whether the pain is "now," "average over the last 24 hours," or "maximum in the past week." Pain intensity assessment should be administered during routine screening and longitudinal monitoring.

Other assessment tools may be useful for special circumstances, such as in the pediatric and dementia settings. ${ }^{8-11}$ Multidimensional pain assessment tools such as the McGill Pain Questionnaire and Brief Pain Inventory are more suitable for use in the research setting than in the clinical setting because of the time requirement and complexity of interpretation.

Table 1 shows how pain characteristics can provide important diagnostic clues to the underlying etiology of the pain. For instance, a patient with neuropathic pain may describe it as burning and radiating to the extremities. Temporal onset may 
Table 1. Pain Types and Characteristics

\begin{tabular}{|lcc|}
\hline \multicolumn{1}{c|}{ Table 1. Pain Types and Characteristics } & \multicolumn{1}{c}{ Other Features } \\
\hline $\begin{array}{l}\text { Somatic nociceptive pain } \\
\text { Bone metastases }\end{array}$ & Quality of Pain & Site specific \\
Wound & Achy, stabbing, throbbing, squeezing, tender, and/or deep & Bone metastases may worsen with movement \\
Mucositis & & Vague and difficult to locate \\
$\begin{array}{l}\text { Visceral nociceptive pain } \\
\text { Liver metastases }\end{array}$ & Sharp, stabbing, squeezing, crampy, and/or gnawing & Referred pain \\
$\begin{array}{l}\text { Bowel obstruction } \\
\text { Coronary ischemia }\end{array}$ & \\
$\begin{array}{l}\text { Urinary retention } \\
\text { Seuropathic pain }\end{array}$ & Burning, shooting, tingling sensation, numbness, scalding; & Radiation along nerve distribution \\
Radiculopathy & may be associated with allodynia and/or hyperalgesia & \\
$\begin{array}{l}\text { Peripheral neuropathy } \\
\text { Post-mastectomy/thoracotomy pain }\end{array}$ & & \\
Phantom limb pain & & \\
\hline
\end{tabular}

help distinguish treatment-related causes from other etiologies. The etiology of acute or chronic pain needs to be investigated thoroughly, because it may be a sign of disease progression. For instance, new onset back pain in a patient with progressive metastatic disease may be the first sign of impending spinal cord compression. Back pain that worsens with recumbency, coughing, or bowel movement may further increase the index of suspicion. ${ }^{12}$

Cancer pain may be persistent, episodic, or both. It is important to assess how many breakthrough pain episodes are present, if any, and what the triggers are. A breakthrough pain episode associated with movement is called incident pain and can be associated with significant functional limitation. ${ }^{13}$ It is also a marker of pain that is more difficult to control. ${ }^{14}$ A good understanding of previous pain relief regimens and the level of adherence can help clinicians tailor their treatment recommendations and the level of patient education needed.

\section{PAIN MECHANISM}

Successful management of pain involves identification of the likely source(s). The etiology for pain can be classified into three major sources in patients with cancer: cancer related (93\%), therapy related (21\%), and unrelated to cancer or its treatments (2\%). ${ }^{15}$ Progressive cancer may result in tissue damage and/or nerve injury through various mechanisms such as compression, infiltration, obstruction, and fracture. Cancer treatments such as surgery (eg, postmastectomy and post-thoracotomy pain), systemic therapy (eg, oxaliplatin-induced peripheral neuropathy, enterocolitis), and radiation (eg, mucositis, dermatitis) may also contribute to increased nociception. Finally, patients with cancer may experience various acute and chronic pain syndromes related to etiologies other than cancer, such as sprains, osteoarthritis, and fibromyalgia. Proper identification of the etiology of pain can inform pain management. For instance, bone pain secondary to progressive cancer may be treated with opioids, steroids, and antineoplastic therapies; bone pain related to spondylosis can usually be managed with acetaminophen alone.

\section{PAIN EXPRESSION AND MULTIDIMENSIONAL ASSESSMENT}

Another important principle in pain assessment involves understanding the pain expression pathway. Figure 1 shows how pain generated as a result of tissue injury is transmitted via afferent pathways, perceived in the somatosensory cortex, and subsequently expressed by the patient. Clinically, pain expression is the only outcome that can be assessed. One implication of this pathway is that tumor progression and tissue damage do not universally translate into physical pain. For some patients, pain expression may be much lower than expected on the basis of radiologic findings. For others, emotional distress related to worsening cancer may contribute more to the pain expression than the tumor mass.

Insights into the pain expression pathway informs a paradigm shift in how clinicians personalize pain assessment and management. We now understand that symptom expression can be modulated by multiple factors, including mood state, chemical coping, and cognition. Proper assessment of these modulating factors is essential for appropriate pain management. For instance, a patient with cancer who has a 9/10 abdominal pain may have visceral pain secondary to progressive liver metastases. In another patient with cancer who has 9/10 abdominal pain, the increased pain expression may be related more to delirium secondary to opioid toxicity and hyperalgesia (Fig 2). Appropriate pain management in the first patient would involve increasing the opioid dose. In contrast, it would be prudent to rotate opioid and manage the delirium rather than further escalating the opioid dose in the second patient, which could paradoxically result in worsening pain expression.

Dame Cicely Saunders coined the term "total pain" in the 1960s to describe the complex interplay between physical, emotional, and existential suffering. ${ }^{16}$ Mood disorders are common in patients with cancer and include adjustment disorders, depression, and anxiety. ${ }^{17}$ Indeed, psychological factors are important modulators of pain expression. Poorly controlled pain could contribute to anxiety and depression; in turn, anxiety and depression may exacerbate pain expression. In patients with advanced cancer, physical pain expression 


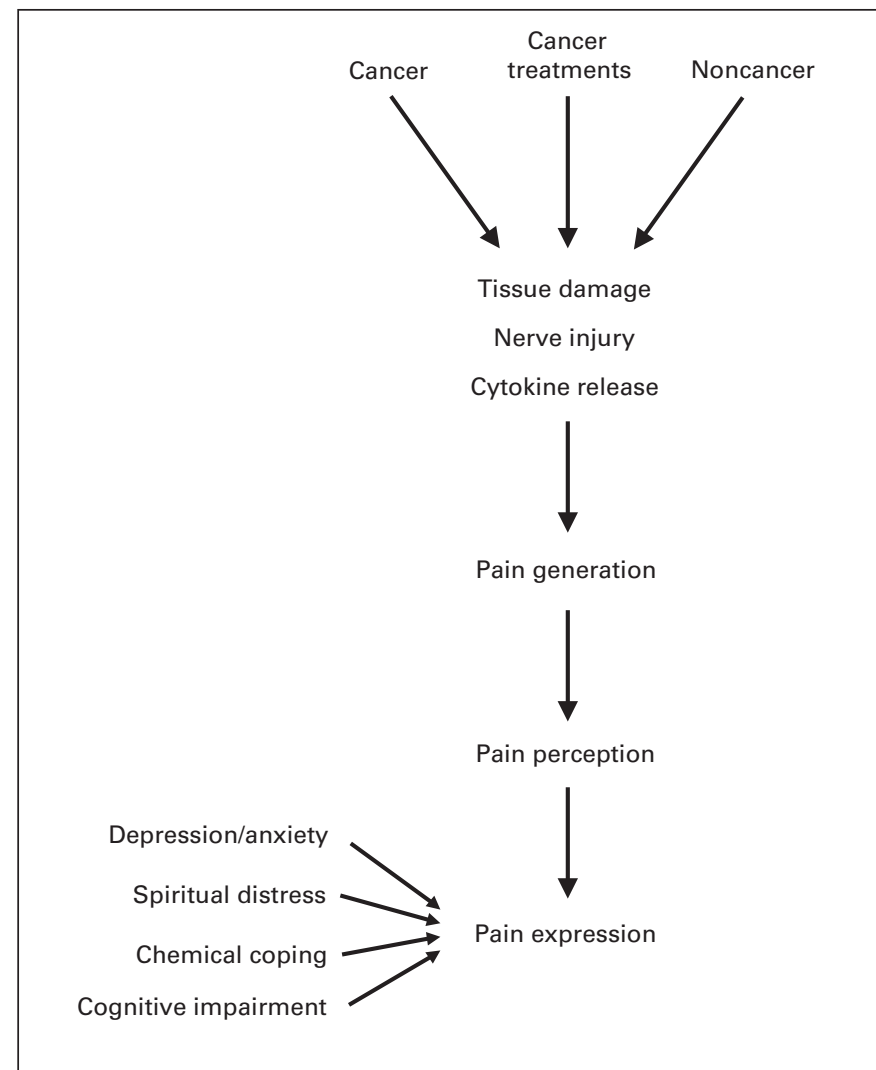

Fig 1. The pain expression pathway. Progressive cancer, cancer treatments, and nonmalignant pathologies can all contribute to tissue injury and inflammation, leading to generation of pain signals peripherally. These signals are then transmitted through afferent pathways and perceived in the somatosensory cortex. The level of pain perceived is further filtered before it is expressed. Multiple modulators may increase or decrease the level of pain expression, including depression, anxiety, spiritual distress, chemical coping, and cognitive impairment. Importantly, we cannot reliably measure pain generation and perception. Pain expression can only be assessed in clinical practice with the use of patient-reported outcomes.

is consistently higher among those with anxiety. ${ }^{18}$ Furthermore, patients with advanced cancer who have spiritual distress reported higher levels of physical pain. ${ }^{19}$

Somatization is a term used to describe the production of recurrent and multiple medical symptoms with no discernible organic cause. ${ }^{20}$ In a large cross-sectional survey of 2,180 noncancer patients, those with chronic low back pain were more likely to have somatization $(14.9 \% v 8.3 \%)$, depression $(13.7 \% v 8.5 \%)$, and anxiety $(9.5 \% v$ $6.2 \%)$ than patients without low back pain. ${ }^{21}$ The term chemical coping has been used to describe the use of opioids to cope with emotional distress and is characterized by an inappropriate and/or excessive use of opioids.

In addition to psychological distress, a history of alcoholism may also contribute to an increased pain expression and a higher propensity to opioid use. At our Supportive Care Clinic, we routinely use the CAGE (Cut down, Annoyed, Guilt, Eye opener) questionnaire for alcoholism screening. ${ }^{22}$ In a consecutive cohort of 665 patients with advanced cancer, 100 (17\%) of 665 were found to be CAGE positive. Patients with a positive CAGE had higher expression of pain (6v 4; $P<.005)$ and were more likely to require opioids (47\% $v 29 \%$ ) compared with patients with a negative CAGE. ${ }^{23}$ Furthermore, among patients with head and neck cancer who completed radiation,

\section{Patient 1}

45-year-old man with stage IV pancreatic cancer and 9 of 10 abdominal pain score

\section{Patient 2}

45-year-old man with stage IV pancreatic cancer and 9 of 10 abdominal pain score

Tumor compression

Delirium

Chemical coping

Tolerance

Psychological distress
Tumor compression

Delirium

Chemical coping

Tolerance

Psychological distress

++++
-
-
-
+

++
+++
-
+
+

Fig 2. Personalized management based on pain modulators. Two patients with the same level of pain expression may have different modulators. In patient 1 , increased nociception as a result of tumor compression is the dominant factor contributing to pain, and an increase in the dose of analgesia may help alleviate the pain. This is in contrast to patient 2, in whom delirium and hyperalgesia from opioid-induced neurotoxicity is contributing to an increased pain expression. For this patient, prompt opioid rotation, rather than increasing the dose of opioid, would be the most appropriate management. This example highlights the importance of recognizing pain modulators and targeting treatment recommendations based on these predictors.

CAGE positivity was associated with prolonged opioid use (9 $v 3$ months; hazard ratio, 2.5). ${ }^{24}$

Interestingly, nicotine use was also associated with increased pain expression and CAGE positivity. ${ }^{25}$ Smokers were also more likely to use opioids, require higher opioid doses, and have more difficulty stopping opioid therapy compared with nonsmokers. ${ }^{26-28}$ Indeed, patients with a history of addiction required higher doses of opioids and longer duration to achieve pain control. ${ }^{14}$ These individuals need to be monitored closely with short intervals between appointments. The goal of therapy should be to optimize functional status rather than to focus on pain relief.

Delirium is a complex neuropsychiatric syndrome characterized by global cognitive dysfunction and is particularly common in patients with cancer in the last weeks of life. ${ }^{29}$ Medications such as opioids and benzodiazepines often contribute to delirium. ${ }^{30}$ As a result of disinhibition, patients with delirium may develop emotional lability and increased symptom expression. ${ }^{31}$ As indicated earlier, for patients with delirium and severe pain, the appropriate management is to treat the delirium with opioid rotation instead of escalating the opioid dose, which could potentially contribute to worsening delirium.

Given the high prevalence of many of these modulators, routine screening for psychological distress (eg, numeric rating scales for anxiety, depression, spiritual distress), alcoholism (eg, CAGE questions), and delirium (eg, Memorial Delirium Assessment Scale) should be part of a comprehensive pain assessment. The Data Supplement includes a sample of the pain assessment forms routinely used in our Supportive Care Clinic.

Our increased understanding of the molecular aberrations implicated in the pathogenesis of cancer has allowed us to develop highly specific therapies targeting these mutations, resulting in more effective treatments with lower toxicities. Similarly, personalized pain management is formulated on the basis of appropriate identification of symptom modulators, previous pain treatments, and also a personalized pain goal (Fig 3). Patients with psychological distress would benefit from counseling, which could result in significant pain improvement and lower doses of opioids. ${ }^{32}$ Successful reversal of delirium may 


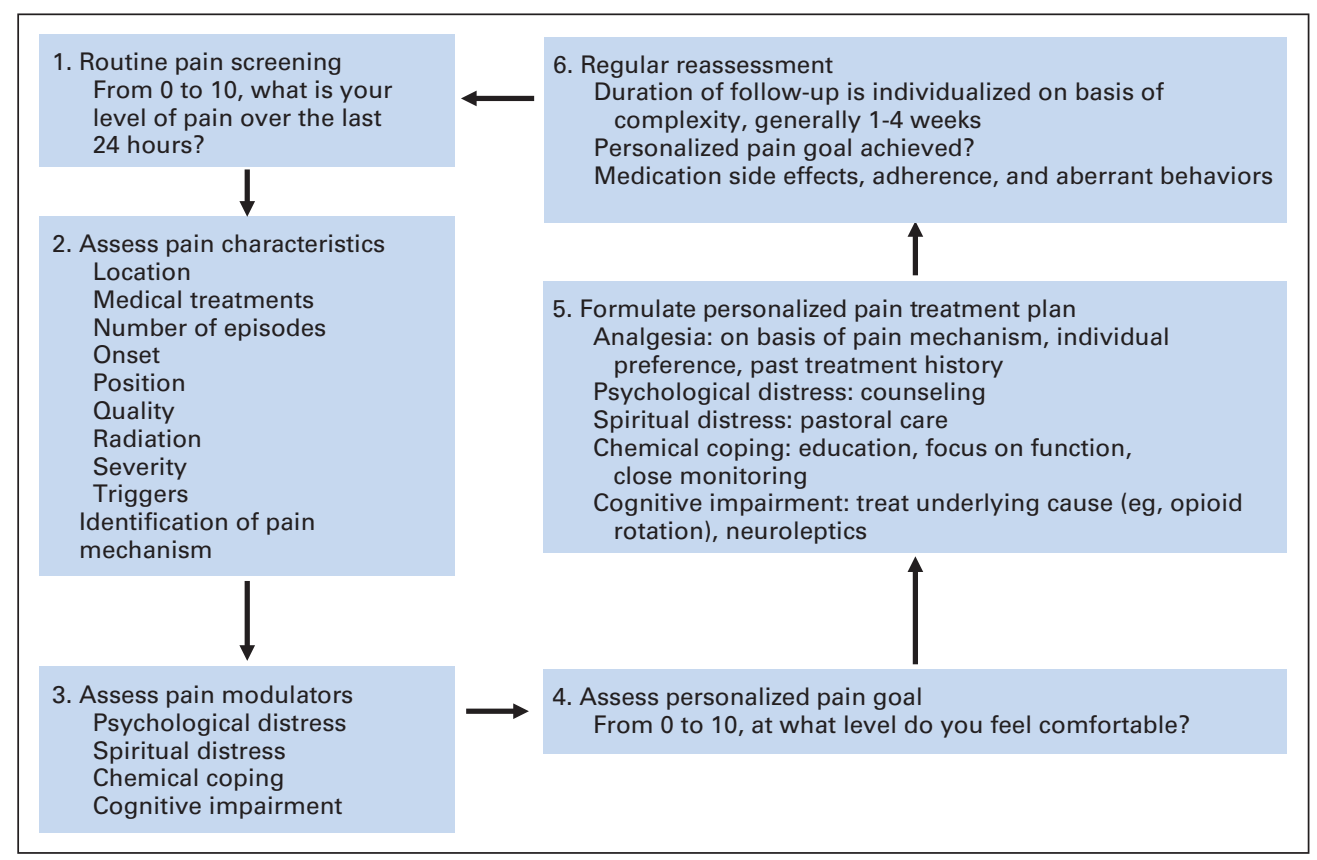

Fig 3. Personalized pain assessment and management. Personalized pain management involves systematic screening, detailing of pain characteristics, identification of potential modulators, documentation of a personalized pain goal, formulation of a multidimensional treatment plan, and longitudinal monitoring. improve pain expression. ${ }^{31}$ Because multiple modulators may be present, interprofessional intervention is essential.

\section{PERSONALIZED PAIN GOAL}

In the era of personalized medicine, pain management may be tailored to the individual need by use of a personalized pain goal (PPG). PPG can be obtained by asking a patient to identify the maximal intensity of pain from 0 to 10 ( 0 , no pain; 10 , worst pain) that would still be considered comfortable.

In a study of 465 patients with advanced cancer seen at our Supportive Care Clinic for consultation, the median PPG was 3 (interquartile range, 2 to 3$).{ }^{33}$ Interestingly, only $2 \%$ of patients reported a PPG of 0 , suggesting that a vast majority of patients were realistic in regard to what can be achieved with contemporary pain therapies. Importantly, PPG remained stable over time. ${ }^{33}$

PPG provides a cutoff to define a personalized response to pain management, in which pain score at or below PPG is defined as a response. On the basis of this criterion, $63 \%, 44 \%$, and $27 \%$ of our patients with mild, moderate, and severe pain achieved a personalized response in their follow-up visit. ${ }^{33}$ This is in contrast to the traditional criteria for defining pain response (ie, reduction in pain by $\geq 2$ of 10 points or $\geq 30 \%$ decrease from baseline), in which $33 \%$, $40 \%$, and $60 \%$ of patients with mild, moderate, and severe pain had a clinical pain response. By using the PPG criteria as a gold standard, clinical pain response criteria had a sensitivity of $52 \%$ and specificity of $98 \%$ for mild pain and a sensitivity of $98 \%$ and specificity of $54 \%$ for severe pain. ${ }^{33}$ Further studies are needed to determine whether achievement of PPG is associated with improved quality of life and to identify predictors of PPG intensity and PPG response.

\section{PREDICTIVE FACTORS FOR CANCER PAIN CONTROL}

Psychological distress, CAGE positivity, and cognitive impairment are not only modulators of pain expression but are also predictive markers for poor pain control. Several pain classification systems have incorporated these modulators, along with other predictive factors, to help clinicians identify patients with pain syndromes that are difficult to control. The first such predictive tool was the Edmonton Staging System (ESS), which includes seven risk factors for poor pain control (Table 2). ${ }^{34}$ On the basis of the total score at presentation, patients were divided into three risk groups: stage I, good risk; stage II, intermediate risk; and stage III, poor risk. In the initial study, 18 (82\%) of 22 with stage I, four (50\%) of eight with stage II, and two (10\%) of 22 with stage III were able to achieve good pain control. This model was subsequently validated in a multicenter cohort of 276 patients and was found to have a sensitivity of $93 \%$ and specificity of $46 \%$ for predicting pain control by 21 days. Thus, the ESS was highly accurate in identifying patients who were able to achieve good pain control, but it has a high false-positive rate for poor pain control. ${ }^{35}$

Further refinements were made to improve the definitional clarity, usability, and accuracy of the ESS, leading to the revised Edmonton Staging System (rESS), which was later renamed the Edmonton Classification System for Cancer Pain (ECSCP). ${ }^{36}$ In a validation study of 746 patients, the five items in rESS were associated with longer time to pain control, greater number of modalities to achieve stable pain control, and higher morphine equivalent daily dose. ${ }^{37}$ The inter-rater reliability ranged from 0.67 to 0.95 . In a secondary analysis, moderate to severe pain expression ( $\geq 4$ of 10 ) at baseline was also found to be significantly associated with duration to achieve stable pain control $(P<.001)$, higher final opioid doses $(P<.001)$, and more adjuvant modalities $(P=.015){ }^{14}$

Huang et $\mathrm{a}^{38}$ have also developed a Cancer Pain Prognostic Scale (CPPS) to predict the likelihood of pain relief in 14 days for patients with moderate to severe pain. Multivariable logistic regression identified four variables associated with pain control, including pain severity, Functional Assessment of Cancer Therapy-General (FACT-G) emotional well-being, initial morphine equivalent daily dose, and mixed pain syndrome. CPPS was predictive of pain control at weeks 1 and 2 but not by week 3 . 


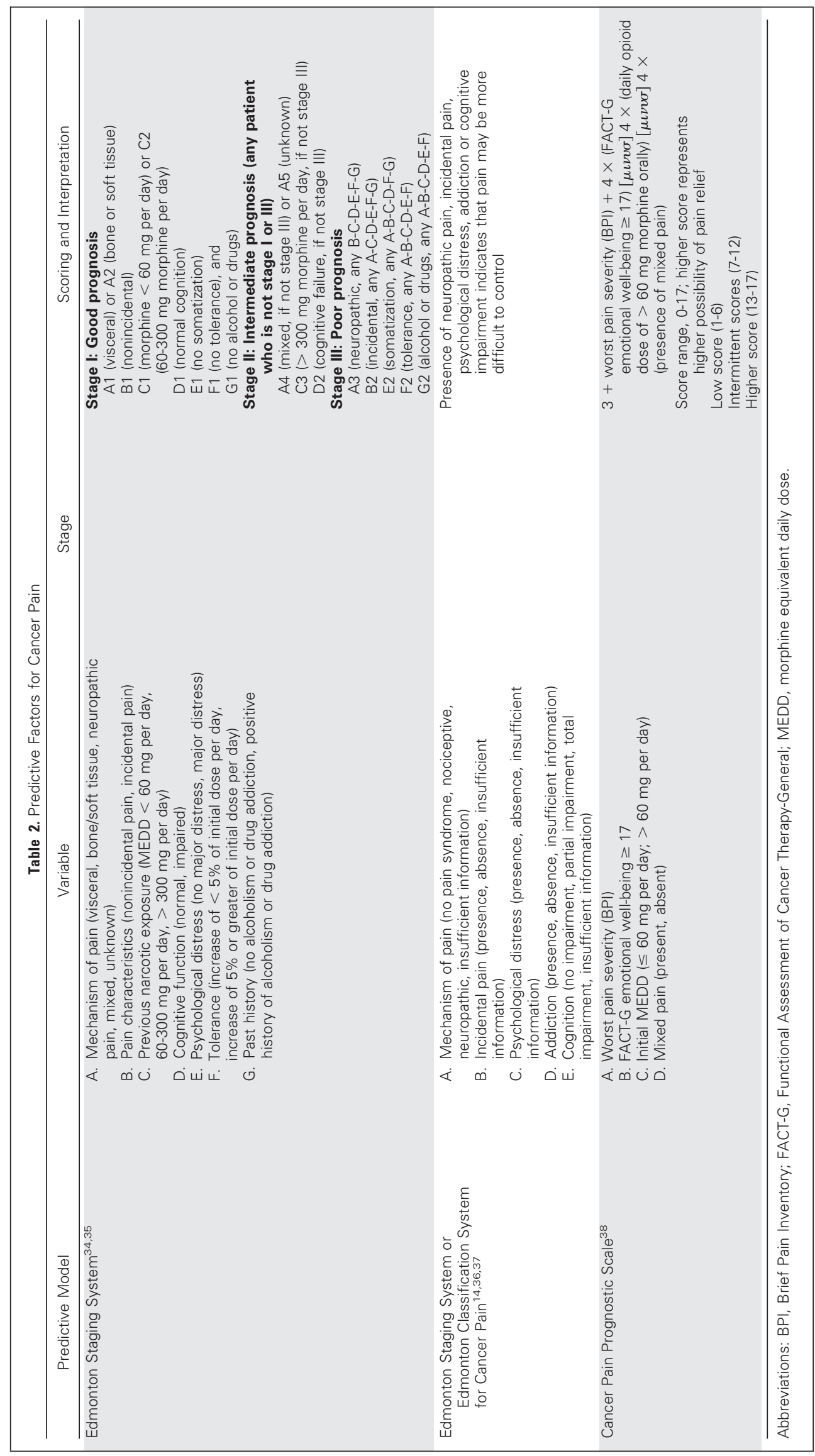


One of the key challenges to the use of these tools is the need for skilled assessments. For instance, in a recent international study of eight countries, the frequency of addictive behavior ranged between $0 \%$ and $50 \%$, with an average of $4.5 \%$ overall. ${ }^{39}$ This wide variation suggests heterogeneity in assessments and potential under-reporting in some centers. ${ }^{23,40,41}$

One practical application of these pain prediction tools is that they may help oncologists and primary care clinicians identify patients with more difficult-to-control pain syndromes; such individuals may benefit from earlier referral to palliative care and/or pain specialists. Further research is needed to improve the accuracy of these predictive tools and to examine whether the use of these tools has a positive impact on pain management.

\section{LONGITUDINAL ASSESSMENTS}

In addition to a comprehensive assessment at initial presentation, successful pain assessment requires longitudinal follow-up, dose titration, and proactive management of adverse effects. The duration between follow-up visits should be customized on the basis of the patients' level of pain expression, pain modulators, and any associated symptoms and comorbidities and may vary from every few days for patients requiring intensive monitoring to every few months for patients with good symptom control and low care needs. The average frequency for follow-up is every 3 to 4 weeks at our Supportive Care Clinic. Patients on strong opioids should be seen at least every 4 weeks for close monitoring and prescription renewal.

Among 1,612 patients seen at our Supportive Care Clinic for a follow-up visit within 30 days of consultation, those who initially presented with no pain experienced an average increase of pain intensity by 2.2 points at follow-up, and those with mild pain initially had an average increase of pain intensity by 0.9 points. In contrast, patients who initially presented with moderate pain experienced an average decrease of pain intensity by 0.4 points, and those with severe pain had an average decrease of pain intensity by 2.5 points. ${ }^{42}$ The change in pain intensity is likely related to a combination of changing disease course, cancer treatments, pain treatments, and other comorbidities. Thus, clinicians should exercise constant vigilance, not only for patients with moderate to severe pain but also for those who had limited pain expression initially because they are at risk of developing pain over the course of their disease.

In addition to assessing pain intensity, it is prudent to assess any associated symptoms such as insomnia, fatigue, anxiety, and depression at follow-up visits. Symptom assessment batteries such as the Edmonton Symptom Assessment System (ESAS) provides comprehensive and rapid assessment of multiple concerns. ${ }^{43}$

For patients who were prescribed opioids, it is important to inquire about the pattern of medication use, such as how many breakthrough doses were used per day on average and whether the patients were taking long-acting medications as prescribed. The rate of adherence to opioid therapy was more than $90 \%$ among patients seen at our Supportive Care Clinic, ${ }^{44}$ which can be attributed to our emphasis on patient education, close monitoring, interdisciplinary management, and a phone support program. Opioid deviation (ie, $<70 \%$ or $>130 \%$ of prescribed dose) was more frequently observed in males and nonwhites. ${ }^{44}$
Monitoring of opioid-related adverse effects such as constipation, nausea and vomiting, and drowsiness is important. Opioidinduced neurotoxicities, including myoclonus, hallucination, and delirium, may develop with high doses of opioids. ${ }^{45}$ Proactive management may improve adherence. In addition to adverse effects, the clinician should assess any barriers to use of pain medications such as fear of opioid addiction and financial factors.

Clinicians should be aware of risk factors for opioid misuse and aberrant behaviors suggestive of opioid abuse. The key risk factors for opioid misuse are younger age (ie, 45 years or younger), a personal history of substance abuse, mental illness, legal problems, and a family history of substance abuse. ${ }^{46}$ Aberrant behaviors concerning potential opioid misuse include, but are not limited to, frequent unsanctioned dose escalations, insistence on specific opioids, concurrent alcoholism and illicit drug use, recurrent loss of prescriptions, lack of follow-up, injection of oral formulations, obtaining forged prescriptions, and selling drugs obtained with a prescription. ${ }^{47}$ Detailed documentation, close monitoring, and referral to a pain specialist are recommended. In a study of patients with head and neck cancer, 63\% were taking opioids at 3 months after completion of their radiation, and 33\% were still taking opioids at 6 months. ${ }^{24} \mathrm{CAGE}$ positivity was a risk factor for prolonged opioid use. ${ }^{24}$

In conclusion, personalized cancer pain management begins with systematic screening, followed by comprehensive pain assessment (Fig 3). Careful characterization of pain informs its etiology and mechanism to guide treatment choices. Identification of modulators such as depression, anxiety, CAGE positivity, and delirium allow clinicians to further target treatments. Documentation of a personalized pain goal provides an individualized response criterion. Finally, longitudinal monitoring tailored to the individual's needs allows us to improve adherence and symptom control. Systematic reviews to further evaluate how different components of cancer pain assessment can improve clinical outcomes would be useful. For instance, the role of electronic diaries for pain assessment and pain clinical pathways need to be evaluated in detail. ${ }^{48,49}$ Insights into the mechanism of pain and the pain expression pathway will allow us to further refine our treatment options.

\section{AUTHORS' DISCLOSURES OF POTENTIAL CONFLICTS OF INTEREST}

Although all authors completed the disclosure declaration, the following author(s) and/or an author's immediate family member(s) indicated a financial or other interest that is relevant to the subject matter under consideration in this article. Certain relationships marked with a " $U$ " are those for which no compensation was received; those relationships marked with a " $C$ " were compensated. For a detailed description of the disclosure categories, or for more information about ASCO's conflict of interest policy, please refer to the Author Disclosure Declaration and the Disclosures of Potential Conflicts of Interest section in Information for Contributors. Employment or Leadership Position: None Consultant or Advisory Role: None Stock Ownership: None Honoraria: None Research Funding: David Hui, Depomed Expert Testimony: None Patents: None Other Remuneration: None

\section{AUTHOR CONTRIBUTIONS}

Conception and design: All authors

Manuscript writing: All authors

Final approval of manuscript: All authors 


\section{REFERENCES}

1. van den Beuken-van Everdingen $M H$, de Rijke $J M$, Kessels AG, et al: High prevalence of pain in patients with cancer in a large population-based study in the Netherlands. Pain 132:312-320, 2007

2. Solano JP, Gomes B, Higginson IJ: A comparison of symptom prevalence in far advanced cancer, AIDS, heart disease, chronic obstructive pulmonary disease and renal disease. J Pain Symptom Manage 31:58-69, 2006

3. Teunissen SC, Wesker W, Kruitwagen C, et al: Symptom prevalence in patients with incurable cancer: A systematic review. J Pain Symptom Manage 34:94-104, 2007

4. Grond S, Zech D, Schug SA, et al: Validation of World Health Organization guidelines for cancer pain relief during the last days and hours of life. J Pain Symptom Manage 6:411-422, 1991

5. Jadad AR, Browman GP: The WHO analgesic ladder for cancer pain management: Stepping up the quality of its evaluation. JAMA 274:1870-1873, 1995

6. Fisch MJ, Lee JW, Weiss M, et al: Prospective, observational study of pain and analgesic prescribing in medical oncology outpatients with breast, colorectal, lung, or prostate cancer. J Clin Oncol 30:1980-1988, 2012

7. Swarm R, Abernethy AP, Anghelescu DL, et al: Adult cancer pain. J Natl Compr Canc Netw 8:1046-1086, 2010

8. Hicks $C L$, von Baeyer $C L$, Spafford $P A$, et al: The Faces Pain Scale-Revised: Toward a common metric in pediatric pain measurement. Pain 93:173183, 2001

9. Hurley AC, Volicer BJ, Hanrahan PA, et al: Assessment of discomfort in advanced Alzheimer patients. Res Nurs Health 15:369-377, 1992

10. Kovach CR, Weissman DE, Griffie J, et al: Assessment and treatment of discomfort for people with late-stage dementia. J Pain Symptom Manage 18:412-419, 1999

11. Warden V, Hurley AC, Volicer L: Development and psychometric evaluation of the Pain Assessment in Advanced Dementia (PAINAD) scale. J Am Med Dir Assoc 4:9-15, 2003

12. Mehta RS, Arnold RM: Evaluation of spinal cord compression \#237. J Palliat Med 14:361-362, 2011

13. Payne R: Recognition and diagnosis of breakthrough pain. Pain Med 8:S3-S7, 2007 (suppl 1)

14. Fainsinger RL, Fairchild $A$, Nekolaichuk $C$, et al: Is pain intensity a predictor of the complexity of cancer pain management? J Clin Oncol 27:585-590, 2009

15. Caraceni A, Portenoy RK: An international survey of cancer pain characteristics and syndromes: IASP Task Force on Cancer Pain-International Association for the Study of Pain. Pain 82:263-274, 1999

16. Clark D: 'Total pain', disciplinary power and the body in the work of Cicely Saunders, 1958-1967. Soc Sci Med 49:727-736, 1999

17. Derogatis LR, Morrow GR, Fetting J, et al: The prevalence of psychiatric disorders among cancer patients. JAMA 249:751-757, 1983
18. Delgado-Guay $M$, Parsons $H A$, Li $Z$, et al: Symptom distress in advanced cancer patients with anxiety and depression in the palliative care setting. Support Care Cancer 17:573-579, 2009

19. Hui $D$, de la Cruz M, Thorney $S$, et al: The frequency and correlates of spiritual distress among patients with advanced cancer admitted to an acute palliative care unit. Am J Hosp Palliat Care 28:264270, 2011

20. Chaturvedi SK, Peter Maguire G, Somashekar BS: Somatization in cancer. Int Rev Psychiatry 18: 49-54, 2006

21. Bener A, Verjee M, Dafeeah EE, et al: Psychological factors: Anxiety, depression, and somatization symptoms in low back pain patients. J Pain Res 6:95-101, 2013

22. Ewing JA: Detecting alcoholism: The CAGE questionnaire. JAMA 252:1905-1907, 1984

23. Parsons HA, Delgado-Guay MO, El Osta B, et al: Alcoholism screening in patients with advanced cancer: Impact on symptom burden and opioid use. J Palliat Med 11:964-968, 2008

24. Kwon JH, Hui D, Chisholm G, et al: Predictors of long-term opioid treatment among patients who receive chemoradiation for head and neck cancer Oncologist 18:768-774, 2013

25. Dev R, Parsons HA, Palla S, et al: Undocumented alcoholism and its correlation with tobacco and illegal drug use in advanced cancer patients. Cancer 117:4551-4556, 2011

26. Ackerman WE 3rd, Ahmad M: Effect of cigarette smoking on serum hydrocodone levels in chronic pain patients. J Ark Med Soc 104:19-21, 2007

27. Hooten WM, Townsend CO, Bruce BK, et al: The effects of smoking status on opioid tapering among patients with chronic pain. Anesth Analg 108:308-315, 2009

28. John $U$, Alte $D$, Hanke $M$, et al: Tobacco smoking in relation to analgesic drug use in a national adult population sample. Drug Alcohol Depend 85:49-55, 2006

29. Hui D, Reddy A, Palla S, et al: Neuroleptic prescription pattern for delirium in patients with advanced cancer. J Palliat Care 27:141-147, 2011

30. Lawlor PG, Gagnon B, Mancini IL, et al: Occurrence, causes, and outcome of delirium in patients with advanced cancer: A prospective study. Arch Intern Med 160:786-794, 2000

31. Delgado-Guay MO, Yennurajalingam S, Bruera E: Delirium with severe symptom expression related to hypercalcemia in a patient with advanced cancer: An interdisciplinary approach to treatment. J Pain Symptom Manage 36:442-449, 2008

32. Reddy A, Hui D, Bruera E: A successful palliative care intervention for cancer pain refractory to intrathecal analgesia. J Pain Symptom Manage 44: 124-130, 2012

33. Dalal $S$, Hui D, Nguyen $L$, et al: Achievement of personalized pain goal in cancer patients referred to a supportive care clinic at a comprehensive cancer center. Cancer 118:3869-3877, 2012

34. Bruera E, MacMillan K, Hanson J, et al: The Edmonton staging system for cancer pain: Preliminary report. Pain 37:203-209, 1989
35. Bruera E, Schoeller T, Wenk R, et al: A prospective multicenter assessment of the Edmonton staging system for cancer pain. J Pain Symptom Manage 10:348-355, 1995

36. Fainsinger RL, Nekolaichuk CL: A "TNM" classification system for cancer pain: The Edmonton Classification System for Cancer Pain (ECS-CP). Support Care Cancer 16:547-555, 2008

37. Fainsinger $R L$, Nekolaichuk $C L$, Lawlor PG, et al: A multicenter study of the revised Edmonton Staging System for classifying cancer pain in advanced cancer patients. J Pain Symptom Manage 29:224-237, 2005

38. Hwang SS, Chang VT, Fairclough DL, et al: Development of a cancer pain prognostic scale. J Pain Symptom Manage 24:366-378, 2002

39. Nekolaichuk $C L$, Fainsinger $R L$, Aass $N$, et al: The Edmonton Classification System for Cancer Pain: Comparison of pain classification features and pain intensity across diverse palliative care settings in eight countries. J Palliat Med 16:516-523, 2013

40. Poulin C, Webster I, Single E: Alcohol disorders in Canada as indicated by the CAGE questionnaire. CMAJ 157:1529-1535, 1997

41. Bruera E, Moyano J, Seifert $L$, et al: The frequency of alcoholism among patients with pain due to terminal cancer. J Pain Symptom Manage 10:599-603, 1995

42. Kang JH, Kwon JH, Hui D, et al: Changes in symptom intensity among cancer patients receiving outpatient palliative care. J Pain Symptom Manage 46:652-660, 2013

43. Bruera E, Kuehn N, Miller MJ, et al: The Edmonton Symptom Assessment System (ESAS): A simple method for the assessment of palliative care patients. J Palliat Care 7:6-9, 1991

44. Nguyen LM, Rhondali $W$, De la Cruz M, et al: Frequency and predictors of patient deviation from prescribed opioids and barriers to opioid pain management in patients with advanced cancer. J Pain Symptom Manage 45:506-516, 2013

45. Bruera E, Hui D: Palliative care research: Lessons learned by our team over the last 25 years. Palliat Med 27:939-951, 2013

46. Alford DP, Livingston EH: JAMA patient page: Misuse of opioid medication. JAMA 309: 2055, 2013

47. Passik SD, Messina J, Golsorkhi A, et al: Aberrant drug-related behavior observed during clinical studies involving patients taking chronic opioid therapy for persistent pain and fentanyl buccal tablet for breakthrough pain. J Pain Symptom Manage [epub ahead of print June 24, 2010]

48. Brink-Huis $A$, van Achterberg T, Schoonhoven $L$ : Pain management: A review of organisation models with integrated processes for the management of pain in adult cancer patients. J Clin Nurs 17:19862000, 2008

49. Morren M, van Dulmen S, Ouwerkerk J, et al: Compliance with momentary pain measurement using electronic diaries: A systematic review. Eur J Pain 13:354-365, 2009 